\title{
As Percepções de Psicólogos em Relação ao Suicídio: Uma Pesquisa Qualitativa no Cenário Sul Catarinense
}

\author{
Psychologists' Perceptions Regarding Suicide: Qualitative Research from Santa Catarina \\ Southern Scenario
}

\section{Las Percepciones de Psicólogos con relación al Suicidio: Una Investigación Cualitativa en el Escenario Sur Catarinense}

\author{
Vitor Apolinário Malgarezi ${ }^{1}$ \\ Sílvia Batista Von Borowski \\ Rousel Aparecida Zanoni Cesconetto \\ Thaís Wachholz \\ Escola Superior de Criciúma
}

\begin{abstract}
Resumo
O manejo profissional perante a ideação suicida é um desafio complexo e pouco explorado em pesquisas. As estatísticas brasileiras demonstram aumento na incidência e dificuldades nas estratégias de proteção. Diante disso e justificado pelo acordo brasileiro em criar um plano de redução de danos, este estudo tem como objetivo descrever as percepções e as especificidades de psicólogos no acompanhamento de risco de suicídio. Trata-se de um estudo qualitativo e exploratório, em que seis psicólogas com histórico de atendimentos ao fenômeno concederam entrevistas. Com base na análise de conteúdo de tais entrevistas, foram identificados três eixos de análise, a saber: impacto perante o suicídio - a experiência dos profissionais; o aprimoramento técnico-metodológico; e o compromisso ético de divulgação. Como considerações, destacam-se a necessidade de uma maior produção científica relacionada a essa temática, a fim de aperfeiçoar discussões, e as possibilidades de atuação em tal contexto.
\end{abstract}

Palavras-chave: prática profissional, suicídio, prevenção

\begin{abstract}
The professional management of suicidal ideation is a complex and little explored challenge. Brazilian statistics show an increase in the incidence and difficulties in protection strategies. Regarding this and considering the Brazilian agreement to create a damage reduction plan, the current study aims to describe perceptions and specificities of psychologists in the monitoring of suicidal risk. It is a qualitative and exploratory study in which six psychologists with a history of attending to the phenomenon granted interviews. From the analysis of the content of such interviews, three axes of analysis were showed, namely: impact of suicide - the professionals' experience, technical and methodological improvement, and ethical disclosure commitment. Furthermore, we highlight the need for significant scientific production related to this theme, to improve discussions, and the possibilities of acting in such a context.

Keywords: professional practice, suicide, prevention

\section{Resumen}

El manejo profesional frente a la ideación suicida es un desafío complejo y poco explorado en investigaciones. Las estadísticas brasileñas demuestran aumento en la incidencia y dificultades en las estrategias de protección. Frente a eso y justificado por el acuerdo brasileño en crear un plan de reducción de daños, este estudio tiene el objetivo de describir las percepciones y las especificidades del acompañamiento psicológico en el riesgo de suicidio. Se trata de un estudio cualitativo y exploratorio, al que seis psicólogas con expediente de atendimientos al fenómeno concedieron entrevistas. Desde análisis de contenido de tales entrevistas, se les identificaron a tres ejes de análisis, a saber: impacto frente al suicidio - la experiencia de los profesionales; mejoramiento técnicometodológico; y compromiso ético de divulgación. Como consideraciones, se destacan la necesidad de una mayor producción científica relacionada a esa temática, a fin de perfeccionar discusiones, y las posibilidades de actuación en tales contextos.
\end{abstract}

Palabras clave: práctica profesional, suicidio, prevención

${ }^{1}$ Rua Cel. Pedro Benedet, n. 225, sala 501, Centro, Criciúma, SC, CEP 88801-250. E-mail: vitorapmal@gmail.com 


\section{Introdução}

O suicídio pode ser compreendido como um ato intencional, consciente, com o intuito de dar fim à própria vida (World Health Organization, 2012). Necessita de atenção e é configurado como um fenômeno complexo e multifatorial, constituindo um problema de saúde pública, problematizado pela OMS desde 1990, sendo reiterado em 2000 e 2012 como grave. Seus impactos antecedem ao ato e suas implicações vão além da perda de uma pessoa, gerando sofrimento à família e ao grupo social (Nunes, Pinto, Lopes, Enes, \& Botti, 2016; Osmarin, 2015).

O suicídio ocorre em todas as etapas do ciclo vital, sendo o envelhecimento o período de maior ocorrência (Scholosser, Rosa, \& More, 2014). Entretanto dados da OMS (2012/2018) apontam um aumento significativo na população jovem, sendo considerada a terceira causa de mortes nessa faixa etária. Além disso, no Brasil, há um crescimento de $29,5 \%$ do percentual de suicídios nos últimos 25 anos, sendo que o número de suicídios pode ser até $20 \%$ maior, caso seja considerada a parcela que é registrada como acidente ou como causa desconhecida (Bertolote, Botega, \& De Leo, 2011). A subnotificação é tida como elemento dificultador na compreensão da proporção desse fenômeno, limitando a criação de planos de prevenção e intervenções mais eficazes diante das reais taxas de mortalidade por suicídio (Alves \& Cadete, 2014).

A Organização Pan-Americana de Saúde indica o investimento na qualificação dos profissionais que lidam direta e indiretamente com os grupos mais vulneráveis, acolhendo essa demanda e oferecendo amparo nas situações de desequilíbrio emocional como uma das estratégias que pode contribuir significativamente na prevenção e na promoção de saúde. Esse desafio é central e mobiliza o compromisso social com a saúde, envolvendo equipes, grupos de trabalho e distintos profissionais de saúde mental. O Brasil, sendo signatário do Plano de Ação de Saúde Mental (WHO, 2013) e dos objetivos de desenvolvimento sustentável (Organização das Nações Unidas, 2016), comprometeu-se em reduzir 10\% da mortalidade por suicídio até 2020.

Nesse sentido, um estudo revelou o quão difícil é, para profissionais da área médica, identificar e prestar atendimento a pacientes em risco de suicídio. O estudo enfatizou que pelo menos $40 \%$ das pessoas que cometeram suicídio consultaram um clínico geral, no mês anterior, demonstrando a importância de uma formação capaz de amparar o sofrimento e identificar riscos (Ramos \& Falcão, 2011). Além disso, a assistência hospitalar é apontada como central no acolhimento e no encaminhamento para os equipamentos de atendimento em saúde mental da rede pública e instituições conveniadas ao Sistema Único de Saúde (SUS) (Gutierrez, 2014).

Destaca-se que conhecer os fatores de risco e o manejo profissional a partir da experiência pode ser uma importante ferramenta de prevenção, pois ". . . toda ameaça de uma pessoa em situação de vulnerabilidade para o suicídio deve ser levada a sério, mesmo quando pareça falsa ou de caráter manipulador" (Vidal \& Contijo, 2013, p. 109), o que exige maior capacidade de percepção e de sensibilidade singular por parte dos profissionais da saúde para o acolhimento (Abreu, Lima, Kohlrausch, \& Soares, 2010). Além disso, os estudos têm dado ênfase ao campo de enfermagem e saúde pública, em que ainda são parcos os estudos envolvendo o psicólogo como agente propulsor da prevenção e da intervenção nos casos de risco (Fukumitsu, 2014). 
Para aumentar as estratégias de proteção e prevenção, é preciso ampliar a compreensão de como os profissionais têm atuado diante dos riscos, entendendo as formas brasileiras e regionais para lidar com tais desafios. Nesse cenário, dentro de uma equipe multiprofissional, destaca-se a importância do exercício do profissional de psicologia diante dos casos de ideação ou tentativa de suicídio, sendo esse profissional capacitado para acolher, intervir e promover saúde (Botega, 2015; Macedo \& Werlang, 2007). Para além da qualificação profissional, é importante atentar para as especificidades culturais e regionais que atravessam o fenômeno do suicídio, pois essas podem ser indicadoras de intervenções mais assertivas.

Considerando tais reflexões sobre o risco de suicídio e o aumento significativo dos números que envolvem tal ato, este artigo pretende descrever as percepções e as especificidades do acompanhamento psicológico no risco de suicídio, segundo psicólogas do sul de Santa Catarina. A região Sul brasileira é responsável por 23\% do total dos casos de suicídio, apesar de apresentar a menor parcela populacional. Além disso, de modo específico, os índices na região Sul de Santa Catarina, conhecida como região carbonífera, é superior à média nacional (10.83 per 100,000), justificando a necessidade de explorar as percepções dos profissionais perante esse fenômeno (Portella, Moretti, Rosa, Quevedo, \& Simões, 2013).

\section{Método}

O estudo se caracteriza por ser de abordagem qualitativa, de delineamento exploratório, em que o propósito foi conhecer as especificidades e percepções dos profissionais de psicologia diante do fenômeno do suicídio (Sampieri, Collado, \& Lucio, 2013).

\section{Participantes}

Foram escolhidos participantes de acordo com os seguintes critérios de inclusão: psicóloga com registro profissional ativo, atuação profissional com histórico de pacientes com ideação, tentativa e/ou suicídio, atuação no sul de Santa Catarina, com pelo menos um ano de formação e que manifestou o aceite para conceder uma entrevista gravada para o estudo.

As participantes foram contatadas pela técnica bola de neve. Uma psicóloga definida por conveniência aceitou participar do projeto e indicou colegas que estivessem dentro do perfil solicitado. Participaram cinco psicólogas com média de formação de 13 anos. Neste estudo, como forma de preservar a imagem e agradecer pela disponibilidade de participar da pesquisa, os nomes das profissionais foram substituídos por pedras preciosas femininas: Ágata, Safira, Ametista, Turquesa e Esmeralda.

\section{Instrumentos}

Como instrumento de investigação, foram utilizados os seguintes recursos: um questionário sociodemográfico e uma entrevista semiestruturada. As questões estavam associadas ao perfil da profissional investigada e a entrevista procurou evocar as percepções das psicólogas sobre o fenômeno com perguntas como "Qual a sua percepção sobre o suicídio?"; "Conte-me sobre sua experiência com ideação, tentativa e/ou suicídio de pacientes"; e "Qual a sua abordagem nos casos em que atendeu? Quais os elementos e estratégias utilizadas no manejo considerou essenciais?". 


\section{Coleta}

As entrevistas foram realizadas no período de julho de 2017 a julho de 2018, em duas cidades de médio porte do sul de Santa Catarina. Foram seguidos todos os preceitos éticos de pesquisa com seres humanos e a Resolução 466/2012, sendo que o projeto foi analisado e aprovado pelo Comitê de Ética da Escola Superior de Criciúma (Esucri) e pela Plataforma Brasil, por meio do CAAE n. 72251417200005356.

O local de coleta foi definido pelas voluntárias, individualmente. As entrevistas foram gravadas de forma previamente autorizada, por meio do Termo de Consentimento Livre e Esclarecido (TCLE), e posteriormente transcritas para análises. Utilizou-se a técnica de análise de conteúdo de Bardin (2011), constituída de pré-análise, exploração do material e tratamento de resultados. Ou seja, três autores realizaram leituras do material e, individualmente, selecionaram falas consideradas significativas para posterior discussão coletiva. Em grupo, cada fala foi discutida e, quando havia discordância, o quarto autor buscava consenso. A partir dessas falas, foram construídas unidades de sentido, compreendidas aqui como categorias a posteriori diante do fenômeno. Por fim, procedeu-se análise com base no sentido advindo das falas alinhado ao marco teórico proposto.

\section{Resultados e Discussão}

A leitura das entrevistas transcritas propiciou três eixos de análise. O primeiro aborda "o impacto emocional perante a ideação, tentativa e/ou suicídio: a experiência dos profissionais"; o segundo, "aprimoramento teórico"; e o terceiro, "a atuação do psicólogo e seu compromisso ético de divulgação", conforme serão abordados na sequência.

\section{Impacto Emocional perante o Suicídio: A Experiência dos Profissionais}

A experiência de um profissional diante de ideação, tentativa e/ou suicídio é um desafio complexo e que exige um preparo técnico e emocional amplo. Na fala da participante Esmeralda, é possível perceber a experiência de frustração: "Eu me considero fracassada nesse sentido, porque eu encaminhei ela pra grupo na época, tinha um grupo, e eu encaminhei ela e ela nunca mais veio, provavelmente eu queria estabelecer vínculo com ela" (Esmeralda, comunicação pessoal, 20 dez. 2017).

Ao ouvir Safira, tem-se a mesma interpretação: "A minha sensação foi horrível, eu fiquei muito ruim assim" (Safira, comunicação pessoal, 20 dez. 2017). A exacerbação de preocupações, inseguranças e medos diante da perda de um paciente é uma das consequências mais perturbadoras vividas pelos profissionais de saúde e que pode estar relacionada com processo psicoterapêutico, por meio do vínculo e da empatia. O profissional se envolve emocionalmente com a situação, sendo mais frequentes sentimentos de raiva, medo ou culpa diante da perda (Botega, 2015; Routhes, Henriques, \& Correia, 2013).

Além da experiência emocional negativa, como de raiva, culpa e dor, outras expressões afetivas podem aparecer, mesmo que de forma não tão explícita. As considerações feitas pelos profissionais podem ser indicativos de negação ou de racionalização mediante a perda de um paciente. Ou seja, a pessoa procura apresentar um comportamento ou uma explicação coerente, aceitável do ponto de vista moral para a situação vivida. Esse indicativo se 
mostra expresso a seguir, em que a explicação sobre a consolidação do suicídio se apresenta sustentada por fatores indicativos da probabilidade de ocorrência, mesmo Turquesa tendo oferecido os devidos cuidados ao paciente:

Então assim, ó, eu não me senti responsável pelo fato, mas muito entristecida, impotente. Naquele dia, eu não trabalhei, acompanhei o velório inteiro. Várias pessoas da família vieram conversar comigo. Ninguém veio se manifestar de uma forma 'mas como que permitiu... né, pelo contrário, todo mundo entendendo a situação. Era um caso bem grave de Psiquiatria, desde a juventude a pessoa já fazia tratamento, tá? Tinha todo o apoio psíquico possível, eu apoiei tudo o que era possível, mas não teve jeito. Ela fez a ingestão de comprimidos e ficou na UTI muito tempo, nesse período de UTI eu tinha contato com os familiares. (Turquesa, comunicação pessoal, 20 dez. 2017)

No entanto, ao longo de seu discurso, a psicóloga vai utilizar a expressão "vida que segue", repetidas vezes, como forma de convencer a si mesma que não há culpabilidade de sua parte no incidente ocorrido, de que sua vida (e seu trabalho) seguem naturalmente, embora seu comportamento possa indicar o oposto, uma preocupação excessiva com familiares e a manutenção de vínculo com esses.

Então assim, ó. . foi dessa forma, e vida segue, então, depois disso ainda fiz contato com pessoas da família, inclusive não só do núcleo familiar, mas da família extensa e a vida que segue. Aí dentro, uma coisa assim, né, vida que segue, mas, assim, quando existe um caso desse na família a gente tem que chamar atenção para a prevenção. (Turquesa, comunicação pessoal, dez. 2017)

Há uma diferença entre ser responsável pelo tratamento de um paciente suicida e ser responsável por sua vida, como Botega (2015) destaca. E nenhuma pessoa, incluindo os profissionais de saúde mental, podem carregar a responsabilidade total pela vida de uma pessoa potencialmente suicida. Apesar disso, é importante destacar que a forma com que o profissional lida com eventos negativos vai repercutir no modo como ele experimenta essa perda. Matos e Borowski (2019) destacam que a regulação emocional do profissional será determinante para o confronto de seu potencial técnico e das competências para lidar com a situação, preferencialmente, utilizando o isolamento para regular essas demandas negativas, o que pode estar relacionado ao rigor ético do sigilo e às expectativas sociais impostas às psicólogas quanto à ética e à capacidade para lidar com a dor. No entanto o desejo de ajuda na superação da crise suicida deve ser ouvido pelos familiares, aliado à capacidade de acreditar no pedido de socorro e ajuda, por parte das equipes, contribuindo para a articulação de uma rede de proteção.

Acho que uma impotência, o que eu poderia ter feito, poderia ter ajudado. E claro também tem uns que cometem suicídio, naquele momento de tristeza mesmo, né, aconteceu alguma coisa e vai lá no impulso da emoção, no impulso da hora. Então eu acho que, sempre que há um suicídio, é uma frustração assim, a saúde mental falhou mais uma vez. (Safira, comunicação pessoal, 20 dez. 2017)

Nesse sentido, o Ministério da Saúde (Brasil, 2009, p. 13) destaca os avanços alcançados nos últimos anos, mas também reconhece suas limitações quando destaca "a questão do 
financiamento insuficiente do setor saúde, a não-priorização efetiva dos investimentos na atenção básica, a precarização do trabalho em muitos lugares, os modos de se produzir a atenção e gestão". Tais reflexões ampliam o sofrimento dos profissionais diante de questões complexas como o suicídio, potencializando falhas e os impactando no manejo e na articulação dos profissionais.

\section{Aprimoramento Técnico-Metodológico}

A necessidade de constante aprimoramento e de formação em centros especializados que respeitem os campos éticos, teóricos e técnicos da psicologia, como ciência e profissão, está largamente descrita, como no Código de Ética (Conselho Federal de Psicologia [CFP], 2005) e na Resolução n. 10, de 2000. Os autores Guareschi, Reis, Machry e Bennemann (2009) destacam as inserções nos currículos de Psicologia de disciplinas que abordem a saúde e possibilitem reflexões que conduzam para a formação do profissional na atuação dentro do Sistema Único de Saúde (SUS). Com a aprovação das Diretrizes Curriculares Nacionais (DCN), a formação do profissional de Saúde deve contemplar o sistema vigente no país, desde 2004.

Mesmo com essas regulamentações, questões relacionadas à conduta de atendimento dos psicólogos clínicos apareceram. Como quando Ágata afirma:

É uma falta de preparo de profissional, né, sem conhecer, muitas vezes, esses profissionais [referindo-se à equipe multiprofissional de saúde], não conhecem o desenvolvimento humano de fato, as questões. . Se falava o mínimo de suicídio [durante a graduação]. A gente não é preparado para lidar com essas mazelas né, de criança tentando suicídio, de adulto tentando suicídio. (Ágata, comunicação pessoal, 20 dez. 2017)

Uma questão central é o quanto o psicólogo deveria estar preparado para atender demandas de risco e ideação do suicídio como parte do sofrimento humano. Boa parte do trabalho desses profissionais estará ligada a ideias de morte ou perdas importantes. Zana e Kovács (2013) fizeram um estudo qualitativo sobre a prática clínica de três psicólogos no atendimento de pacientes que apresentavam risco de suicídio. As análises destacam o quão conflitantes podem ser esses momentos para o profissional: a questão do sigilo, a implicação no vínculo e a discussão sobre a máxima dos profissionais da saúde de salvar vidas a qualquer custo. Para as autoras, essas implicações impactam no manejo com a situação de risco, que pode ser determinante para preservar a vida ou para gerar a quebra do vínculo. Por outro viés, ampliar o debate acerca dos atendimentos psicoterápicos para a saúde como um todo é fundamental. Ao psicólogo, isso pode representar uma forma de enfrentamento da situação.

Os autores Vidal e Gontijo (2013) discutem a forma de acolhimento vivido por pessoas que tentaram suicídio na rede pública de saúde. Destacaram os discursos, muitas vezes, preconceituosos e violentos, que reforçam o sofrimento e agravam a condição: "A baixa capacitação profissional de atendimento e as deficiências estruturais do serviço e do sistema de saúde como um todo induzem os profissionais desses serviços a se posicionarem de maneira impessoal e com dificuldade de atuação de forma humanizada" (p. 112). Essa constatação é manifestada por Esmeralda, quando argumenta ser "necessário um preparo no hospital pra receber o suicida, né, e pra entender o suicídio como um problema de saúde". 
Nesse sentido, a atuação profissional dentro de um centro de atenção psicossocial articulado para receber a demanda pode ser fundamental para redução dos casos de morte autoprovocada, somando maior adesão quando há um engajamento familiar (Heck et al., 2012). Além disso, há de se considerar o papel hospitalar como espaço de acolhimento e assistência, pois, quando o atendimento é realizado com qualidade, prontidão e segurança, há uma maior adesão e aceitação do paciente ao tratamento (Godim, 2015).

A psicologia pode ocupar um papel fundamental nessa construção de saberes e atuações que corroboram a necessidade de prevenção. A escuta acurada e acolhedora sobre a história de vida do indivíduo, sua percepção de determinados eventos de vida, os significados e as interpretações daquilo que o impactou repercutem num melhor planejamento de ações de prevenção e promoção de saúde. Os psicólogos devem estar atentos aos níveis de sofrimento psicológico que podem ocorrer como resposta à incapacidade de lidar com determinados acontecimentos de vida, podendo constituir um importante fator de proteção aos riscos (Sobrinho \& Campos, 2016).

Essa reflexão pode incluir a equipe multiprofissional, que nem sempre conhece os processos psicológicos do sofrimento e precisa ser acolhida e orientada. Destaca-se o psicólogo como mediador, capaz de ampliar a percepção dos colegas e acolher a dor de quem tenta o suicídio. No entanto acredita-se que é na formação profissional que a mudança precisa acontecer, incluindo no currículo disciplinas que problematizem a violência autoprovocada e ampliem a capacitação dos profissionais para mediar a orientação desses casos. Portanto isso vai além do acolhimento, o profissional de psicologia pode ser um mecanismo efetivo nas equipes multiprofissionais, o que pode ser visto como a construção de uma percepção mais abrangente do papel desse profissional no contexto de saúde, superando a ausência de espaços de discussão e de clareza quanto aos procedimentos a serem seguidos diante do risco (Reis \& Faro, 2016; Zana \& Kovács, 2013).

O constante aperfeiçoamento possibilita a garantia de direitos individuais, permite capacitar os profissionais para novas demandas e contribui para a diminuição do sofrimento psíquico do sujeito, aumentando sua autonomia e qualidade de vida. Assim, é desejada "uma capacitação, um curso de especialização. Enfim, algo que desse conta desse assunto" (Safira, comunicação pessoal, 20 dez. 2017).

\section{A Atuação do Psicólogo e seu Compromisso Ético de Divulgação}

Esta categoria aborda um tema importante para todos os profissionais da saúde, destacando-se os psicólogos. A resolução do CFP n. 010/05 estabelece o Código de Ética, que, já em seus princípios fundamentais, afirma que o psicólogo deve promover a universalização do acesso da população a informações, serviços, padrões éticos e conhecimento da ciência psicológica (CFP, 2005).

As práticas de divulgação e publicidade estão devidamente dispostas na resolução do CFP n. 010, de 1997, em que são permitidas somente práticas ou técnicas devidamente reconhecidas pelos critérios científicos estabelecidos, exceto as que estejam em processo de pesquisa, sendo que a não observância dessa resolução constitui uma infração passível de pena. Essas questões foram recorrentes nas participantes, como destacado por Turquesa ". . . a importância do papel do psicólogo em divulgar mais as informações acerca do nosso 
trabalho nesses casos ou nos demais é de necessidade pública" (Turquesa, comunicação pessoal, 20 dez. 2017).

Há uma preocupação dos profissionais com esse trabalho de divulgação, visando à promoção dos saberes psicológicos, o que contribui para um processo de desmistificação da própria profissão, conforme destacado por Pereira (2008) e Dimenstein (2001). "É preciso que seja realizada essa divulgação de uma forma que chegue em mais número de pessoas, né" (Ágata, comunicação pessoal, 20 dez. 2017). Atingindo esse maior contingente populacional, tendo como base a Lei n. 8.080 (Brasil, 1990), é possível promover atendimentos e encaminhamos visando à promoção, à proteção e à recuperação da saúde.

Entender que tipo de divulgação pode repercutir em um apoio a colegas psicólogos e quais podem auxiliar no acolhimento dos casos que não chegam por falta de informação é factual para construção de caminhos. O fazer da psicologia perante esse fenômeno em Santa Catarina não difere do restante do país, e a articulação da rede para atender a essa demanda é fundamental, como destaca Ametista:

Eu acho que seria adequado tu ter uma equipe que atenda, que ele questiona aqui, você precisa de uma equipe que seja referência pra isso, que seja qualificada para conduzir. Então teria que ter um centro de referência para essas situações de risco. (Comunicação pessoal, 20 dez. 2017)

Numa perspectiva de promoção de saúde, toda a rede de profissionais deve ser corresponsável pelo cuidado integral das pessoas. Desde a Lei 8.080/90, incita-se o fomento ao desenvolvimento de estratégias que possam impactar em qualidade de vida. Isso inclui, efetivamente, a educação e a informação de acesso. É necessário articular os profissionais e criar procedimentos capazes de deixar claros os papéis e as formas de acolhimento.

\section{Considerações Finais}

Como reflexões importantes advindas deste estudo, destacam-se o investimento em uma formação capaz de amparar práticas mais efetivas. Embora o Código de Ética Profissional do Psicólogo, em seu artigo $9^{\circ}$, oriente a categoria sobre a possibilidade de quebra de sigilo em situações que envolvam riscos, e compreenda-se a ideação suicida como tal, a forma de condução de uma situação dessa natureza não está contemplada no documento, o que pode gerar insegurança e medo quanto ao tipo de abordagem que deve ser feita.

Diante da realidade de saúde brasileira, discutir o "adequado" é outro fundamento básico para novas e mais eficazes formas de acolher e prevenir a violência autoprovocada, como problematiza a WHO (2012). Sugerem-se estudos que possam descrever práticas de intervenção nas diferentes regiões brasileiras, a fim de ampliar a troca de experiências entre profissionais da psicologia, quiçá, exemplos de articulação e fortalecimento de uma rede de proteção entre profissionais da saúde. Além da qualificação profissional, é importante atentar para as especificidades culturais e regionais que atravessam o fenômeno do suicídio, como formas de indicadores para intervenções mais assertivas.

Em relação às limitações desta pesquisa, menciona-se dentro da sua metodologia o número de participantes. Apesar da riqueza das reflexões e do sentido da experiência compartilhada ter sido significativo, pesquisas quantitativas podem gerar dados mais fidedignos 
quanto ao sofrimento e às dificuldades vividas por essa categoria profissional. Outro fator que pode ter interferido nos dados é a cultura da profissão de psicologia em não ferir o sigilo de informações clínicas e/ou teorizar como forma de se proteger da exposição em uma pesquisa. A prática da psicologia é muito solitária, especialmente na clínica, e compartilhar experiências pode ser um campo desconhecido para as voluntárias que aceitaram participar, ainda que se tenha garantido o anonimato. Por fim, ampliar o número de pesquisas quanto ao contexto de atuação dos psicólogos diante de práticas que geram desconforto, como a ideação, tentativa e/ou suicídio, pode repercutir em apoio e estratégias benéficas à saúde emocional desses profissionais.

\section{Referências}

Abreu, K. P., Lima, M. A. D. S., Kohlrausch, E., \& Soares, J. F. (2010). Comportamento suicida: fatores de risco e intervenções preventivas. Revista Eletrônica de Enfermagem, 12(1), 195-200. Disponível em http://fen.ufg.br/revista/v12/n1/pdf/v12n1a24.pdf

Alves, M. A. G, \& Cadete, M. M. M. (2015). Tentativa de suicídio infanto-juvenil: Lesão da parte ou do todo? Ciência \& Saúde Coletiva, 20(1). Disponível em http://www.scielo.br/ scielo.php?pid=S1413-81232015000100075\&script=sci_arttext\&tlng=pt

Bardin, L. (2011). Análise de conteúdo. São Paulo: Edições 70.

Bertolote, J. M., Botega, N., \& De Leo, D. (2011). Inequities in suicide prevention in Brazil. The Lancet, 378(9797). 1137. Disponivel em https://www.thelancet.com/journals/lancet/ article/PIIS0140-6736(11)61502-X/fulltext

Botega, N. J. (2015). Crise suicida. Porto Alegre: Artmed.

Brasil. Lei n. 8.080, de 19 de setembro de 1990. Dispõe sobre as condições para a promoção, proteção e recuperação da Saúde, a organização e o funcionamento dos serviços correspondentes e dá outras providências. Brasília, 1990 [e suas atualizações]. Disponível em http://www.planalto.gov.br/ccivil_03/leis/L8080.htm

Brasil. (2009). Ministério da Saúde. Secretaria de Atenção à Saúde. Política Nacional de Humanização da Atenção e Gestão do SUS. Brasília: Ministério da Saúde. [Redes de produção de saúde. Série B. Textos Básicos de Saúde]. Disponível em http://bvsms.saude. gov.br/bvs/publicacoes/humaniza_sus_atencao_basica.pdf

Conselho Federal de Psicologia. (1997). Resolução n. 010, de 20 de outubro de 1997. Disponível em https://site.cfp.org.br/wp-content/uploads/1997/10/resolucao1997_10. pdf

Conselho Federal de Psicologia. (2005). Resolução n. 010, de 21 de julho de 2005. Código de Ética Profissional do Psicólogo. Disponível em https://site.cfp.org.br/wp-content/ uploads/2012/07/codigo-de-etica-psicologia.pdf

Dimenstein, M. (2001). O psicólogo e o compromisso social no contexto da saúde coletiva. Psicologia em Estudo, 6(2), 57-63. Disponível em http://www.scielo.br/pdf/\%0D/pe/ v6n2/v6n2a08.pdf

Fukumitsu, K. O. (2014). O psicoterapeuta diante do comportamento suicida. Psicologia USP, 25(3), 270-275. Disponível em http://www.scielo.br/pdf/pusp/v25n3/0103-6564pusp-25-03-0270.pdf 
Godim, D. S. M. A intervenção da Psicologia: Tentativas de suicídio e urgência hospitalar. (2015) Revista Científica da FMC, 10(2), 12-16. Disponível em http://www.fmc.br/wpcontent/uploads/2016/04/Rev-Cient-FMC-2-2015-12-16-1.pdf

Guareschi, N. M. de F., Reis, C., Machry, D. S., \& Bennemann, T. (2009) A formação em Psicologia e o profissional da Saúde para o SUS (Sistema Único de Saúde). Arquivos Brasileiros de Psicologia, 61(3), 35-45. Disponível em http://pepsic.bvsalud.org/scielo. php?script=sci_arttext\&pid=S1809-52672009000300005

Gutierrez, B. A. O. (2014). Assistência hospitalar na tentativa de suicídio. Psicologia UPS, 25(3), 262-269. Disponível em http://www.scielo.br/pdf/pusp/v25n3/0103-6564pusp-25-03-0262.pdf

Heck, R. M., Kantorski, L. P., Borges, A. M., Lopes, C. V., Santos, M. C., \& Pinho, L. B. (2012). Ação dos profissionais de um centro de atenção psicossocial diante de usuários com tentativa e risco de suicídio. Texto \& Contexto - Enfermagem, 21(1), 26-33. Disponível em http://www.scielo.br/pdf/tce/v21n1/a03v21n1

Macedo, M. M. K., \& Werlang, B. S. G. (2007). Tentativa de Suicídio: O traumático via ato-dor. Psicologia: Teoria e Pesquisa, 23(2) 185-194. Disponível em http://www.scielo.br/pdf/ ptp/v23n2/a09v23n2.pdf

Matos, M. S., \& Borowski, S. B. V. (2019). Vivências emocionais e estratégias de regulação emocional de psicólogos clínicos: Um estudo qualitativo. Estudos Interdisciplinares em Psicologia, 10(3), 160-180. Disponível em http://srv-009.uel.br/revistas/uel/index.php/ eip/article/view/32047/26649

Nunes, F. D. D., Pinto, J. A. F., Lopes, M., Enes, C. L. E., \& Botti, L. (2016). O fenômeno do suicídio entre os familiares sobreviventes: Revisão integrativa. Revista Portuguesa de Enfermagem de Saúde Mental, 15, 17-22. Disponível em http://www.scielo.mec.pt/scielo. php?script=sci_arttext\&pid=S1647-21602016000100003

Organização das Nações Unidas (2016). Objetivos de Desenvolvimento Sustentável (ODS). Disponível em http://www.itamaraty.gov.br/images/ed_desenvsust/20160119-ODS.pdf

Osmarin, M. S. A. (2015). Suicídio: O luto dos sobreviventes. Conselho Federal de Psicologia (pp. 45-58). Brasília: CFP. Disponível em http://www.psicologia.pt/artigos/textos/A0981. pdf

Pereira, M. C. S. (2008). O mundo contemporâneo e o compromisso de psicólogos com a definição de uma nova estética da vida social. In A. F. Silveira et al. (Orgs.), Cidadania e participação social (pp. 35-43). Rio de Janeiro: Centro Edelstein de Pesquisas Sociais, 2008. Disponível em http://books.scielo.org/id/hn3q6/pdf/silveira-9788599662885-04. pdf

Portella, C. H., Moretti, G. P., Rosa, M. I., Quevedo, J., \& Simões, P. W.T. A. (2013). Epidemiological profile of suicide in the Santa Catarina Coal Mining Region from 1980 to 2007. Trends in Psychiatry and Psychotherapy, 35(2), 128-133. Disponível em http://www.scielo.br/scielo. php?script=sci_arttext\&pid=S2237-60892013000200006\#correspondence

Ramos, I. N. B., \& Falcão, E. B. M. (2011). Suicídio: Um tema pouco conhecido na formação médica. Revista Brasileira de Educação Médica, 35(4), 507-516. Disponível em http:// www.scielo.br/pdf/ rbem/v35n4/a10v35n4.pdf 
Reis, B.A. O., \& Faro, A. (2016). Aresidência multiprofissional eaformação do psicólogo da saúde: Um relato de experiência. Revista Psicologia e Saúde, 8(1), 62-70. Disponível em http:// pepsic.bvsalud.org/scielo.php?script=sci_arttext\&pid=S2177-093X2016000100008

Routhes, I. A., Henriques, M. R., \& Correia, R. S. (2013). Suicídio de um paciente: A experiência de médicos e psicólogos portugueses. Revista Portuguesa de Saúde Pública, 31(2), 168-178. Disponível em https://www.sciencedirect.com/science/article/pii/S087090251300031X Sampieri, R., Collado, C., \& Lucio, M. P. (2013). Metodologia de pesquisa. Porto Alegre: Penso. Scholosser, A., Rosa, G. F. C., \& More, C. L. O. O. (2014). Revisão: Comportamento suicida ao longo do ciclo vital. Temas em Psicologia, 22(1), 133-145. Disponível em http://pepsic. bvsalud.org/scielo.php?script=sci_arttext\&pid=S1413-389X2014000100011

Sobrinho, A. T., \& Campos, R. C. (2016). Percepção de acontecimentos de vida negativos, depressão e risco de suicídio em jovens adultos. Análise Psicológica, 34(1), 47-59. doi: http://dx.doi.org/10.14417/ap.1061

Vidal,C.E.L., \&Gontijo, E.D. (2013).Tentativas desuicídioeoacolhimentonosserviços deurgência: A percepção de quem tenta. Cadernos Saúde Coletiva, 21(2), 108-114. Disponível em http:// www.scielo.br/scielo.php?pid=S1414-462X2013000200002\&script=sci_abstract\&tlng=pt

World Health Organization. (2012). Public health action for the prevention of suicide: A framework. Disponivel em https://apps. who.int/iris/bitstream/handle/10665/75166/9789241503570_eng. pdf;jsessionid=D51F94C2984ECAB0416C458BE4865B73? sequence $=1$

World Health Organization (2013). Mental Health Action Plan 2013-2020. Disponível em http://www.mhinnovation.net/sites/default/files/downloads/resource/MHAP.pdf

Zana, A. R. O.; Kovacs, M. J. (2013). O psicólogo e o atendimento a pacientes com ideação ou tentativa desuicídio. Estudose Pesquisasem Psicologia,13(3), 897-921. Disponível em http:// pepsic.bvsalud.org/scielo.php?script=sci_abstract\&pid=S1808-42812013000300006

Recebido em: 07/09/2018

Última revisão: 29/05/2019

Aceite final: 11/06/2019

\section{Sobre os autores:}

Vitor Apolinário Malgarezi - Acadêmico de Psicologia. E-mail: vitorapmal@gmail.com, Orcid: http://orcid.org/0000-0003-4596-9539

Sílvia Batista Von Borowski - Mestre em Psicologia Clínica pela Universidade do Vale do Rio dos Sinos (Unisinos), RS. MBA em Gestão Educacional e Docência do Ensino Superior. Psicóloga clínica. E-mail: silviavon@esucri.com.br, Orcid: http://orcid.org/0000-0002-4706-5966

Rousel Aparecida Zanoni Cesconetto - Acadêmica de Psicologia. E-mail: selzinha@gmail.com, Orcid: http://orcid.org/0000-0001-9979-1647

Thaís Wachholz - Mestre em Educação pela Universidade do Estado de Santa Catarina (UNESC). Docente do ensino superior. Psicóloga clínica. E-mail: thaispsicanalista@gmail.com, Orcid: http://orcid.org/0000-0001-5330-4161 
